\title{
PERSPECTIVAS DE DIAGNÓSTICO SOBRE LA DISTRIBUCIÓN DEL LIDERAZGO EN ORGANIZACIONES ESCOLARES: UN ANÁLISIS EN DOS DIMENSIONES CLAVE
}

\author{
Oscar Maureira ${ }^{1}$, Sergio Garay ${ }^{2}$, Luis Ahumada ${ }^{3}$ y \\ Carlos Ascencio ${ }^{4}$
}

\begin{abstract}
RESUMEN
El estudio del liderazgo escolar se ha desarrollado como área relevante, pertinente y consistente en la explicación de mejora de la eficacia escolar. Enfoques de liderazgo instructivo, transformacional y para aprendizajes han caracterizado ámbitos clave de práctica directiva. Sin embargo, dada la naturaleza de las organizaciones escolares (definidas más por la interacción que por la acción individual), emerge la perspectiva distribuida de liderazgo. El siguiente trabajo, de carácter descriptivo analítico, aporta fundamentos teóricos y operacionales respecto de cómo aproximarse al liderazgo distribuido para su diagnóstico escolar. Se estudian separadamente, por medio de análisis factoriales exploratorios, dos dimensiones de un cuestionario que forma parte de un estudio mayor, administrado a 1.518 directivos y docentes de dos muestras incidentales de 100 escuelas y liceos de Chile. Los resultados constatan la existencia de un solo factor que explicaría la brecha entre la percepción actual y deseada de la distribución de liderazgo, en tanto que sobre patrones de distribución, solo tres de las seis escalas utilizadas serían unifactoriales. Se concluye que la primera dimensión es robusta y constituye una estrategia plausible para un diagnóstico inicial, dadas las limitaciones de deseabilidad social que tiene tanto el constructo como el instrumento.
\end{abstract}

Conceptos clave: dimensiones de liderazgo distribuido, escalas de distribución del liderazgo, liderazgo distribuido.

\section{DIAGNOSTIC PERSPECTIVES ON THE DISTRIBUTION OF LEADERSHIP IN SCHOOL ORGANIZATIONS: AN ANALYSIS ON TWO KEY DIMENSIONS}

\section{ABSTRACT}

The study of school leadership has evolved as a relevant, meaningful and consistent area, when it comes to explaining improvements in school effectiveness. Instructional and transformational leadership, as well as leadership for learning approaches, have characterized key areas of a school director's practice. However, given the nature of school organizations, whereby interaction

1 Universidad Católica Silva Henríquez, Santiago, Chile. Contacto: omaureir@ucsh.cl

2 Universidad Católica Silva Henríquez, Santiago, Chile. Contacto: sgaray@ucsh.cl

3 Pontificia Universidad Católica de Valparaíso, Valparaíso, Chile. Contacto: luis.ahumada@pucv.cl

4 Universidad Católica Silva Henríquez, Santiago, Chile. Contacto: cascenciog@ucsh.cl 
rather than individual action plays a major role in building influential capacities, the perspective of distributed leadership has emerged as a distinctive feature. Hence, this work, of a descriptiveanalytical nature, provides theoretical and operational foundations on how to approach distributed leadership for its diagnosis in schools. Two dimensions of a questionnaire from a larger study, administered to 1.518 directors and teachers of two incidental samples of one hundred elementary and high schools in Chile, are analyzed separately by exploratory factor analysis. As the main results of the study, the existence of a single factor that would narrow the gap between the current and desired perception of the leadership distribution is verified. Regarding distribution patterns, only three of the six scales that comprise it, would be unifactorial. As a conclusion, the first dimension is robust and constitutes a viable strategy for an initial diagnosis, considering the limitations of social desirability that both the construct and the instrument may have.

Key concepts: distributed leadership, distributed leadership dimensions, leadership distribution scales.

\section{Introducción}

En los diferentes estudios e investigaciones acerca de liderazgo en las organizaciones escolares ha predominado la perspectiva del liderazgo directivo, centrado principalmente en las manifestaciones individuales de influencia de quien detenta el poder posicional en la estructura organizativa. Sin embargo, la alta interacción que se genera por las múltiples actividades formativas y organizativas escolares (consejos de profesores, reuniones de directivos docentes, departamentos, apoderados, etc.) devela un número no menor de instancias colectivas de comunicación en las que participan agentes educativos y pedagógicos que interactúan en, con y para la formación de los estudiantes. En este sentido, focalizar y reducir el análisis de influencias al comportamiento directivo de un director o directora supone una omisión importante de conocimiento. Por ello, progresivamente diversas investigaciones (Ahumada, 2010; Bennett, Wise, Woods \& Harvey, 2003; Bolden, 2011; Bolívar, 2012; Gronn, 2002; Harris, 2012; López-Yáñez, 2012; Spillane, 2006) justifican la pertinencia y relevancia del estudio del liderazgo escolar como un fenómeno colectivo más que unipersonal, dado que este sería clave como conocimiento para desarrollar capacidades escolares de mejoramiento y sustentabilidad para los aprendizajes, especialmente en atención al incremento de capital social y decisional. Además, los múltiples componentes que lo conforman, así como las relaciones 
166 PERSPECTIVAS DE DIAGNÓSTICO SOBRE LA DISTRIBUCIÓN DEL LIDERAZGO EN ORGANIZACIONES ESCOLARES: UN ANÁLISIS EN DOS DIMENSIONES CLAVE - O. Maureira, S. Garay, L. Ahumada y C. Ascencio

con variables de procesos con las que se le asocia dan sentido a la profundización de su estudio y desarrollo.

En la perspectiva de analizar la relación entre liderazgo distribuido y compromiso con la organización escolar, Hulpia, Devos y Van Keer (2011) encontraron que la cooperación y participación de los equipos de trabajo en procesos de toma de decisiones son variables críticas para predecir el compromiso de los profesores. Por su parte, García Torres (2018), en un estudio realizado en Singapur, señala que el liderazgo distribuido predice de manera significativa el desempeño profesional y la satisfacción de los docentes. En este sentido Larraín (2017), en un estudio realizado en ocho establecimientos públicos de EE.UU., reportó que la implementación de un proyecto de liderazgo distribuido permitió observar cambios en la confianza dentro de los equipos de docentes, los cuales se analizaron en las siguientes dimensiones: competencia profesional, integridad, consideración del otro y respeto. Los resultados sugirieron que el desarrollo de liderazgo distribuido puede influir positivamente en la confianza y, consecuentemente, en la enseñanza y aprendizaje escolar.

Igualmente, el estudio en liderazgo transcultural basado en los indicadores del Estudio Internacional sobre la Enseñanza y el Aprendizaje (TALIS) de 2013 (Organisation for Economic Co-operation and Development, OECD, 2014) en Europa (Sans-Martín, Guàrdia y Triadó-Ivern, 2016) destaca que los atributos tanto del liderazgo instructivo (o pedagógico) como del distribuido son características distintivas que influyen positivamente en la colaboración y el desempeño escolar de las instituciones.

En Chile, investigaciones basadas en casos múltiples de mejora escolar (Bellei, Morawietz, Valenzuela y Vanni, 2015) y de sus trayectorias (Bellei, Valenzuela, Vanni y Contreras, 2014) develan que las duplas constituidas por directores y jefes de Unidades Técnicas Pedagógicas (UTP) son determinantes en el desarrollo de la mejora escolar sostenible. De este modo - y asociado a la normalización del funcionamiento escolar luego del terremoto de 2010 en Chile-, el estudio de casos de liderazgo en tiempos de crisis descrito por Pascual, Larraguibel, Zenteno y Guarda (2016) en dos liceos en la 
Región del Maule, señala como clave la distribución ordenada de liderazgos, pues en uno de los casos se evidenció cómo el director se apoyó en un extenso equipo directivo (compuesto por 14 personas, entre docentes y asistentes de la educación), mientras que en el otro liceo estudiado fue el equipo directivo el que distribuyó el liderazgo entre los docentes, específicamente, en los jefes de departamento, a fin de organizar el normal funcionamiento del establecimiento.

Por su parte, desde la gestión curricular, Derby (2017) indagó profundamente en la relación entre el liderazgo distribuido y el liderazgo instruccional en tres escuelas municipales, usando como referente teórico el enfoque distribuido basado en Hulpia, Devos y Rosseel (2009) y el análisis de medidas de centralidad, reciprocidad y densidad de redes sociales, concluyendo que a la hora de abordar el tema de gestión curricular prevalecía un alto grado de centralidad, un carácter unidireccional y una baja densidad en las relaciones. Esto, en resumen, significa que a lo sumo dos personas recibían la mayor cantidad de requerimientos, constatándose una débil reciprocidad en la relación.

En una perspectiva de investigación acción colaborativa, Ahumada, Castro y Maureira (2018) llevaron a cabo un estudio en 20 escuelas y liceos en la Región de Valparaíso, en el cual se buscó fortalecer el liderazgo distribuido sobre la base del modelo de Harris (2014), enfocándose en el aumento de capacidades en los aprendizajes organizacionales y el desarrollo profesional docente y de los estudiantiles.

Otro estudio pertinente acerca de la distribución del liderazgo, aunque más orientado a la explicación a partir de una metodología de ecuaciones estructurales, es el realizado por López y Gallegos (2017) acerca de la incidencia del liderazgo distribuido y de los desempeños escolares medidos por el Sistema de Medición de la Calidad de la Educación (Simce) de Matemática en cerca de 70 escuelas destacadas entre sus similares. Allí se concluye que las dimensiones de participación y cooperación del liderazgo distribuido, desde el enfoque de Hulpia et al. (2009), se asocian positivamente con un aumento de 12,7 y 13,8 puntos respectivamente en el 
puntaje promedio en la prueba Simce de Matemática. Por su parte, un reciente estudio comparativo en escuelas del norte de Chile realizado por Rojas-Andrade, Prosser y Bonilla (2018), cuyo propósito fue comparar la distribución del liderazgo en escuelas de logro intermedio e inicial en el Simce, señala que dos establecimientos de logro intermedio, respecto de aquellos de logro inicial, obtuvieron puntajes significativamente superiores en las escalas de visión escolar, cultura escolar, programa instruccional, uso de artefactos, liderazgo docente y relaciones intuitivas de trabajo, elementos constitutivos de la escala de liderazgo distribuido de Davis (2009), la que se empleó como referente de medición.

Desde las políticas educativas, cada vez más se busca una mayor autonomía y responsabilidad por los resultados alcanzados por los directivos docentes, lo cual queda en evidencia a través de mecanismos como los convenios de desempeño (Ley No 20.501, 2011). Así mismo, las características de la organización —definidas en el contexto progresivo de las instalaciones de los Servicios Locales Educativos (SLE) - emergen como una modalidad de desarrollo de capacidades intra e interescolares del aprendizaje en red y representan uno de los componentes de la estrategia de mejoramiento del liderazgo distribuido. En este sentido aplicado como una práctica más que como un estilo (Ahumada, González y Pino, 2016), ello implica un proceso de influencia que actúa sobre cambios de creencias y delegación de tareas en actores que conforman dicha red con un propósito de mejora. No obstante, Montecinos, Aravena y Tagle (2016) señalan que existen debilidades en el trabajo de equipo en las escuelas y en las condiciones para llevarlo a cabo, lo que se transforma en un obstáculo importante para impulsar un liderazgo que se haga cargo de la colaboración y, preferentemente, de la corresponsabilidad de la calidad de los aprendizajes.

El presente estudio pretende facilitar una mayor comprensión - preferentemente para las audiencias profesionales- de las principales perspectivas tanto teóricas como prácticas acerca de la distribución del liderazgo escolar, con el objeto de favorecer el diagnóstico y/o la reflexión en torno a este enfoque. 
Dadas las múltiples variables afines al desarrollo de capacidades de innovación y mejora asociadas al liderazgo distribuido, en este estudio se optó por focalizar el análisis en solo dos dimensiones. La primera de ellas se refiere a la percepción acerca de la situación actual y deseada de la distribución del liderazgo, dimensión considerada útil para dar cuenta de los puntos más críticos. La segunda dimensión, en tanto, aborda distintas modalidades o patrones de distribución del liderazgo, los que dan cuenta de los diversos procesos organizativos. Esta dimensión es ventajosa para comprender cómo se distribuye el liderazgo en la escuela o liceo, así como también para avanzar hacia su implementación en la cultura del establecimiento, evolucionando hacia la consolidación de un liderazgo distribuido independiente de los cargos y de los líderes formales de la organización. Al respecto, el estudio aporta con una herramienta concreta para identificar el fenómeno y los ámbitos en los cuales se puede abordar.

La investigación optó por la adaptación de la escala de Pont, Nusche y Moorman (2009), pues ofrece una perspectiva práctica para propiciar la reflexión en la comunidad escolar al interrogar acerca del nivel de percepción actual y deseada, ayudando a visibilizar las brechas tanto agregadas (total escala) como desagregadas (ítems) que podrían, eventualmente, activar el inicio de acciones para la mejora en aspectos específicos, o bien, en lo global. De igual modo, se privilegió la perspectiva de MacBeath (2011), sobre patrones de distribución de liderazgo, dado que en su origen es una investigación cualitativa sustantiva y exhaustiva en provisión de categorías (o estadios) que consideró el diálogo conjunto de investigadores y docentes de 11 instituciones, tanto de educación infantil como primaria y secundaria, que fueron recomendadas como ejemplos de buenas prácticas de distribución de liderazgo por parte de autoridades locales en Inglaterra. Este esfuerzo derivó en la generación de un conjunto de indicadores (ítems) por cada estadio, que contribuyen a identificar la predominancia de patrones de distribución de liderazgo en las instituciones educativas inglesas.

Dada su relevancia, pertinencia y consistencia en la explicación de varios procesos y resultados de mejora e innovación escolar, los argumentos que sugieren que el liderazgo distribuido es una perspectiva 
170 PERSPECTIVAS DE DIAGNÓSTICO SOBRE LA DISTRIBUCIÓN DEL LIDERAZGO EN ORGANIZACIONES ESCOLARES: UN ANÁLISIS EN DOS DIMENSIONES CLAVE - O. Maureira, S. Garay, L. Ahumada y C. Ascencio

plausible para comprender la práctica del liderazgo en las escuelas son múltiples y diversos. En este sentido, el siguiente trabajo —-más allá de fundamentar y sustentar este foco de práctica— tiene por propósito describir y aportar con dimensiones e indicadores de distribución de liderazgo que permitan a las comunidades educativas realizar un diagnóstico para impulsar eventualmente su desarrollo, dada su estrecha relación con las trayectorias escolares de mejora e innovación.

\section{Principales fundamentos y atributos de la concepción del liderazgo distribuido}

De tiempo en tiempo emergen acontecimientos relevantes en el desarrollo teórico en los que se quiebra una visión y surgen nuevas miradas que logran interpretar, de manera renovada, un fenómeno connatural en los grupos humanos: este es el caso del liderazgo de las organizaciones en general y, particularmente, en las instituciones escolares. En efecto, iniciado el siglo XXI, el desarrollo teórico y práctico de un modelo de liderazgo en el que se destacan Spilllane y MacBeath, se ha concentrado en una visión compartida en el contexto de una comunidad educativa (MacBeath, 2005; Spillane, Halverson \& Diamond, 2001; 2004). Esta perspectiva viene a decantar el proceso que se inició con las primeras y antiguas teorías de los rasgos, hasta lo que hoy se discute y propone como lo más "renovado" del desarrollo teórico en este campo: el fenómeno de la distribución del liderazgo. En este tránsito, se ha contado con muchas aproximaciones y con cada una de ellas también se ha renovado la definición del fenómeno.

Sin embargo, existe convicción en cuanto a que asistimos a un cambio sustancial en la comprensión del liderazgo como un fenómeno de influencia que, en palabras de Fernández y Zambrano (2018), se puede entender como una articulación de arquetipos y fortalezas del ser y la efectividad en un tejido social. Con tal fundamento se intenta superar la visión persistente y centrada en la figura del líder solitario, heroico, de capacidades infinitas e inagotables, para pasar al liderazgo que se ejerce en el espacio de las relaciones sociales.

Respecto de las características del liderazgo distribuido, este tiene su origen (López, 2013) en los principios de las teorías de la 
cognición distribuida (Hutchins, 1995) y de la actividad (Engeström, 2001). Sin embargo, dicho enfoque se configura también a partir de las propuestas del liderazgo compartido (Senge, Ross, Smith, Roberts \& Kleiner, 1995), por lo cual con frecuencia la literatura se refiere indistintamente a liderazgo distribuido o compartido. De esta forma, términos como democrático, delegativo o coliderazgo emergen para connotarlo, por lo que una de las dificultades para comprender el concepto del liderazgo distribuido y poder medirlo radica en la imprecisión de su uso, tanto a nivel semántico como en su interpretación científica. Debido a esto es posible observar más la adjetivación de distribuido que distributivo, aunque la segunda corresponde a un adjetivo propiamente tal y se define, según la Real Academia Española, como que toca o atañe a la distribución (RAE, 2019).

Por otra parte, el concepto de liderazgo distribuido se asocia con dos acepciones (Gronn, 2002). La primera está basada en el concepto de desagregación aditiva, es decir, en función de cuántos roles de liderazgos disponibles existen en la organización para cumplir sus propósitos, similarmente a lo que se ha entendido por densidad del liderazgo. Se reconoce, en este caso, que la dirección escolar requiere múltiples líderes (Gronn, 2002; Sergiovianni, 1984; Spillane, 2006), que asuman responsabilidades de liderazgo en distintas actividades formales e informales (Gronn, 2002; Timperley, 2005). En cambio, la segunda acepción —más holística— comporta una acción coordinada, más que una conducta individual agregada, es decir, la distribución del liderazgo no significa la delegación en los demás de las tareas propias del director o directora, sino una forma de acción colectiva que integra actividades de muchas personas que trabajan para su movilización hacia el cambio y la mejora (Harris citado en Zener, 2011).

De esta forma, como clave para comprender el liderazgo como práctica social en las organizaciones escolares, ciertos autores se centran en las interacciones que se producen entre líderes, seguidores y situación en contextos educativos (Spillane, 2006), particularmente en aquellas que tienen directa influencia en la motivación, conocimientos y emociones de los integrantes de la 
172 PERSPECTIVAS DE DIAGNÓSTICO SOBRE LA DISTRIBUCIÓN DEL LIDERAZGO EN ORGANIZACIONES ESCOLARES: UN ANÁLISIS EN DOS DIMENSIONES CLAVE - O. Maureira, S. Garay, L. Ahumada y C. Ascencio

comunidad escolar, puesto que definir y focalizar el liderazgo excluye las prácticas que no están relacionadas con el trabajo esencial de la organización escolar (Spillane \& Healey, 2010). Una de las cuestiones centrales que se plantea en torno a esta perspectiva distribuida del liderazgo (Spillane, 2006) es que este se construye colectivamente para aportar en conocimientos y significados en la organización, definiéndose como ampliada a través de la práctica, esto es, en las interacciones entre líderes, seguidores y su situación (Spillane, 2006; Spillane, Camburn, Pustejovsky, Stitziel \& Lewis, 2008). Así, se desplaza parcialmente la responsabilidad individual-jerárquica al grupo y se configura el constructo líder-plus, que refiere a la acción e interacción conjunta, tanto de quienes detentan la autoridad formal como de aquellos que emergen como líderes informales en la práctica del liderazgo en un contexto situacional y temporal.

Lo central y esencial de la perspectiva distribuida del liderazgo es la focalización en su práctica (Spillane y Ortiz, citado en Weinstein, 2016), lo que por un lado implica las múltiples interacciones que se producen en la actividad escolar entre diversos agentes educativos $y$, por otro, su carácter social. Sin embargo, un aspecto que media e influye tal práctica es la situación, pues para la perspectiva distribuida del liderazgo este constructo no opera en el vacío (Spillane y Ortiz, citado en Weinstein, 2016). En este sentido, los aspectos que definen la situación en la que ocurre la práctica del liderazgo pueden ser variados, pudiendo ser facilitadores o inhibidores de la interacción entre los agentes educativos.

En relación con los estudios o enfoques que posibilitan alguna aproximación más operacional para la medición del liderazgo distribuido es posible referir la escala que proponen Pont et al. (2009), la cual se configura a partir de 10 ítems que buscan establecer la brecha entre la percepción de lo actual y deseable sobre las oportunidades, promoción y estructuras de desarrollo de liderazgos al interior de los establecimientos, así como la consideración de que el liderazgo compartido favorecería los aprendizajes. La Tabla 1 da cuenta de la adaptación y contextualización de esta dimensión. 
Tabla 1

Escala adaptada de Pont et al. (2009) sobre distribución de liderazgo

\begin{tabular}{|c|c|c|c|c|c|c|c|c|c|c|}
\hline \multicolumn{5}{|c|}{ Situación actual } & \multirow[t]{2}{*}{ Distribución del liderazgo (DL) } & \multicolumn{5}{|c|}{ Sería deseable } \\
\hline$\frac{\pi}{\frac{\pi}{\pi}}$ & \& & 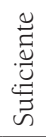 & 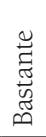 & 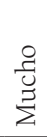 & & $\frac{\pi}{\pi}$ & ஜ & 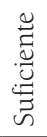 & 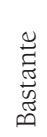 & $\frac{8}{\stackrel{g}{U}}$ \\
\hline 1 & 2 & 3 & 4 & 5 & $\begin{array}{l}\text { Las tareas de liderazgo son } \\
\text { compartidas ampliamente en la } \\
\text { escuela. }\end{array}$ & 1 & 2 & 3 & 4 & 5 \\
\hline 1 & 2 & 3 & 4 & 5 & $\begin{array}{l}\text { Se promueve la participación en } \\
\text { equipos de liderazgo. }\end{array}$ & 1 & 2 & 3 & 4 & 5 \\
\hline 1 & 2 & 3 & 4 & 5 & $\begin{array}{l}\text { Existen estrategias para preparar } \\
\text { líderes que renueven los cargos } \\
\text { directivos. }\end{array}$ & 1 & 2 & 3 & 4 & 5 \\
\hline 1 & 2 & 3 & 4 & 5 & $\begin{array}{l}\text { La rendición de cuentas refleja } \\
\text { un trabajo compartido por la } \\
\text { dirección del establecimiento. }\end{array}$ & 1 & 2 & 3 & 4 & 5 \\
\hline 1 & 2 & 3 & 4 & 5 & $\begin{array}{l}\text { Los integrantes de los organismos } \\
\text { colegiados tienen oportunidad } \\
\text { de desarrollar capacidades de } \\
\text { liderazgo. }\end{array}$ & 1 & 2 & 3 & 4 & 5 \\
\hline 1 & 2 & 3 & 4 & 5 & $\begin{array}{l}\text { Los mandos medios líderes tienen } \\
\text { oportunidades de desarrollo de } \\
\text { liderazgo. }\end{array}$ & 1 & 2 & 3 & 4 & 5 \\
\hline 1 & 2 & 3 & 4 & 5 & $\begin{array}{l}\text { El liderazgo compartido es } \\
\text { reconocido y reforzado en la } \\
\text { política pública existente. }\end{array}$ & 1 & 2 & 3 & 4 & 5 \\
\hline 1 & 2 & 3 & 4 & 5 & $\begin{array}{l}\text { Las estructuras de las escuelas } \\
\text { fomentan el desarrollo de equipos } \\
\text { de liderazgo. }\end{array}$ & 1 & 2 & 3 & 4 & 5 \\
\hline 1 & 2 & 3 & 4 & 5 & $\begin{array}{l}\text { Desarrollar un liderazgo } \\
\text { compartido favorece los resultados } \\
\text { de aprendizaje de los estudiantes. }\end{array}$ & 1 & 2 & 3 & 4 & 5 \\
\hline
\end{tabular}

Fuente: Elaboración propia.

En relación con las modalidades de distribución del liderazgo, estas se configuran a partir de diversos procesos organizativos. La Tabla 2, basada en MacBeath (2011), da cuenta de los patrones para aproximarse al conocimiento de modalidades de distribución del liderazgo en las organizaciones escolares. 
174 PERSPECTIVAS DE DIAGNÓSTICO SOBRE LA DISTRIBUCIÓN DEL LIDERAZGO EN ORGANIZACIONES ESCOLARES: UN ANÁLISIS EN DOS DIMENSIONES CLAVE - O. Maureira,

S. Garay, L. Ahumada y C. Ascencio

Tabla 2

Escala de estadios o patrones de distribución del liderazgo basadas en MacBeath (2011)

\begin{tabular}{|c|c|c|}
\hline $\begin{array}{l}\text { Patrones de } \\
\text { distribución } \\
\text { de liderazgo }\end{array}$ & $\begin{array}{c}\text { Descripción } \\
\text { breve de cada } \\
\text { conceptualización }\end{array}$ & Ítems \\
\hline \multirow{8}{*}{ Formal } & \multirow{8}{*}{$\begin{array}{l}\text { La manera en } \\
\text { que se asignan } \\
\text { responsabilidades } \\
\text { directivas en } \\
\text { estructuras y } \\
\text { roles formales } \\
\text { institucionales. }\end{array}$} & $\begin{array}{l}\text { La estructura organizacional de la escuela } \\
\text { determina con claridad todos los puestos de } \\
\text { liderazgo posible. }\end{array}$ \\
\hline & & $\begin{array}{l}\text { Las capacidades de liderazgo que se deben ejercer } \\
\text { en el establecimiento están descritas claramente en } \\
\text { roles y funciones. }\end{array}$ \\
\hline & & $\begin{array}{l}\text { El ejercicio de liderazgo es coherente con las } \\
\text { normativas que regulan el funcionamiento de la } \\
\text { institución. }\end{array}$ \\
\hline & & $\begin{array}{l}\text { Se asignan formalmente responsabilidades de } \\
\text { liderazgo. }\end{array}$ \\
\hline & & $\begin{array}{l}\text { Existe empoderamiento en roles formales de } \\
\text { liderazgo. }\end{array}$ \\
\hline & & $\begin{array}{l}\text { Existe conciencia del rol de liderazgo en directivos } \\
\text { docentes. }\end{array}$ \\
\hline & & $\begin{array}{l}\text { La estructura jerárquica predomina en la toma de } \\
\text { decisiones. }\end{array}$ \\
\hline & & $\begin{array}{l}\text { El liderazgo en esta institución está explícitamente } \\
\text { reflejado en las estructuras formales. }\end{array}$ \\
\hline \multirow{7}{*}{ Pragmática } & \multirow{7}{*}{$\begin{array}{l}\text { Delegación ad hoc } \\
\text { de carga laboral. Su } \\
\text { característica es la } \\
\text { inmediatez y falta } \\
\text { de visión de largo } \\
\text { plazo en aspectos } \\
\text { de sucesión y } \\
\text { construcción de } \\
\text { capacidades. }\end{array}$} & $\begin{array}{l}\text { Se asignan tareas puntuales de liderazgo ante } \\
\text { necesidades emergentes. }\end{array}$ \\
\hline & & $\begin{array}{l}\text { Se comparten roles de liderazgo en función de } \\
\text { requerimientos emergentes. }\end{array}$ \\
\hline & & $\begin{array}{l}\text { La inmediatez en la asignación de responsabilidades } \\
\text { de liderazgo predomina más que la visión. }\end{array}$ \\
\hline & & $\begin{array}{l}\text { Los docentes eluden responsabilidades de } \\
\text { liderazgo directivo. }\end{array}$ \\
\hline & & $\begin{array}{l}\text { Se asignan responsabilidades específicas de } \\
\text { liderazgo. }\end{array}$ \\
\hline & & $\begin{array}{l}\text { Se asignan tareas de liderazgo en función de } \\
\text { criterios prácticos. }\end{array}$ \\
\hline & & $\begin{array}{l}\text { Se asignan tareas de liderazgo sobre la base del } \\
\text { conocimiento que poseen las personas. }\end{array}$ \\
\hline
\end{tabular}




\begin{tabular}{|c|c|c|}
\hline \multirow{8}{*}{ Estratégica } & \multirow{8}{*}{$\begin{array}{l}\text { Orientación hacia } \\
\text { metas, alineándose } \\
\text { tras un objetivo } \\
\text { de largo plazo } \\
\text { en función del } \\
\text { mejoramiento } \\
\text { escolar. }\end{array}$} & $\begin{array}{l}\text { Se cuenta con una visión clara de mediano y largo } \\
\text { plazo que guía la asignación de roles de liderazgo. }\end{array}$ \\
\hline & & $\begin{array}{l}\text { La toma de decisiones en tareas de liderazgo es } \\
\text { prevista por el alineamiento estratégico. }\end{array}$ \\
\hline & & $\begin{array}{l}\text { Los criterios que guían el desarrollo profesional } \\
\text { docente son de equipos más que individuales. }\end{array}$ \\
\hline & & El liderazgo es de equipo más que de individuos. \\
\hline & & $\begin{array}{l}\text { Se asignan estratégicamente responsabilidades de } \\
\text { liderazgo. }\end{array}$ \\
\hline & & $\begin{array}{l}\text { Se asignan tareas de liderazgo en función de las } \\
\text { metas de mejora escolar. }\end{array}$ \\
\hline & & $\begin{array}{l}\text { En la cultura de esta institución se asume que el } \\
\text { liderazgo está distribuido en las personas como } \\
\text { conjunto. }\end{array}$ \\
\hline & & $\begin{array}{l}\text { Se alinean las capacidades de trabajar en equipo } \\
\text { con el logro de metas estratégicas. }\end{array}$ \\
\hline \multirow{7}{*}{ Incremental } & \multirow{7}{*}{$\begin{array}{l}\text { Suelta el control } \\
\text { de arriba hacia } \\
\text { abajo y amplía } \\
\text { las posibilidades } \\
\text { del potencial del } \\
\text { liderazgo. }\end{array}$} & $\begin{array}{l}\text { La dirección logra detectar con tiempo a los } \\
\text { potenciales líderes que van apareciendo en el } \\
\text { trabajo cotidiano. }\end{array}$ \\
\hline & & $\begin{array}{l}\text { Existe apoyo desde la dirección para potenciar los } \\
\text { nuevos liderazgos que se van manifestando. }\end{array}$ \\
\hline & & $\begin{array}{l}\text { La dirección trabaja en equipo con los docentes a } \\
\text { favor de la institución. }\end{array}$ \\
\hline & & $\begin{array}{l}\text { Existen instancias formativas para desarrollar } \\
\text { capacidades en potenciales líderes. }\end{array}$ \\
\hline & & $\begin{array}{l}\text { Se generan espacios de trabajo y colaboración para } \\
\text { los nuevos líderes que van apareciendo. }\end{array}$ \\
\hline & & La dirección es capaz de "compartir" su liderazgo. \\
\hline & & $\begin{array}{l}\text { Se van asignando tareas de liderazgo en forma } \\
\text { gradual. }\end{array}$ \\
\hline \multirow{8}{*}{ Oportuno } & \multirow{8}{*}{$\begin{array}{l}\text { El liderazgo es } \\
\text { adoptado más } \\
\text { que entregado. Es } \\
\text { asumido más que } \\
\text { otorgado. }\end{array}$} & $\begin{array}{l}\text { Se asumen responsabilidades de liderazgo en } \\
\text { forma oportuna. }\end{array}$ \\
\hline & & Siempre aparece un líder cuando se le necesita. \\
\hline & & El liderazgo es adoptado más que conferido. \\
\hline & & $\begin{array}{l}\text { Existen oportunidades en que se nota la ausencia } \\
\text { de un líder. }\end{array}$ \\
\hline & & $\begin{array}{l}\text { Los líderes formales de la institución son capaces } \\
\text { de enfrentar los desafíos y situaciones emergentes } \\
\text { que los requieran. }\end{array}$ \\
\hline & & Existe un alto nivel de iniciativa para liderar. \\
\hline & & $\begin{array}{l}\text { Se dan oportunidades para ceder liderazgo a } \\
\text { estamentos de la comunidad educativa. }\end{array}$ \\
\hline & & $\begin{array}{l}\text { Se antepone la oportunidad de liderar más que la } \\
\text { planificación y la asignación de roles. }\end{array}$ \\
\hline
\end{tabular}



ORGANIZACIONES ESCOLARES: UN ANÁLISIS EN DOS DIMENSIONES CLAVE - O. Maureira, S. Garay, L. Ahumada y C. Ascencio

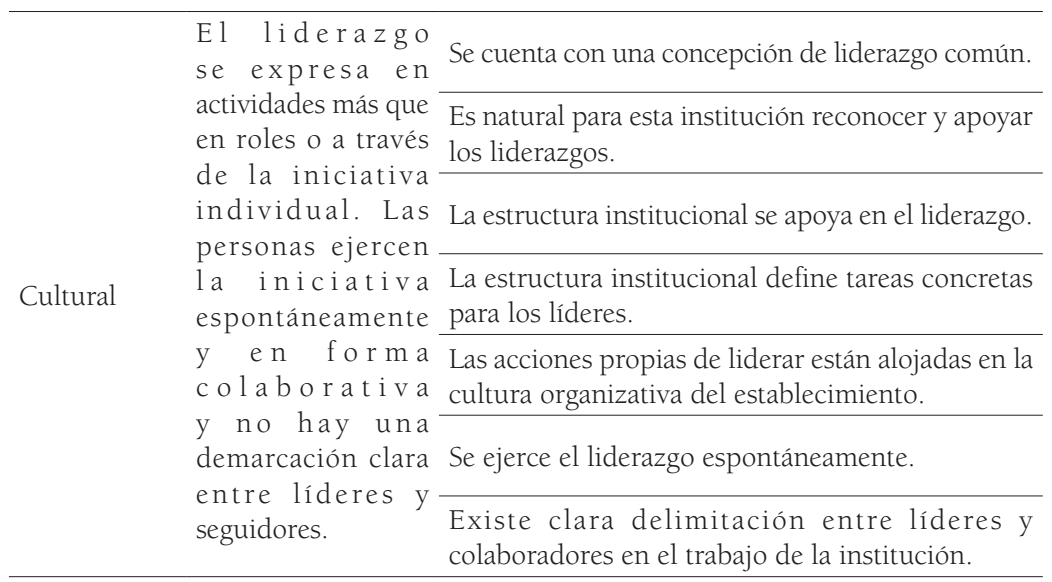

Fuente: Elaboración propia.

De este modo, siguiendo las directrices de la construcción de instrumentos (Morales, Urosa y Blanco, 2003), en el contexto de un primer estudio exploratorio descriptivo sobre liderazgo distribuido en una muestra de 51 escuelas primarias en Chile (Maureira, 2013), se produjo un cuestionario que integró, adaptó y recreó un conjunto de indicadores, agrupados en escalas — las que fueron objeto de análisis a través de una segunda aplicación en una muestra incidental e independiente de la primera,- con el objeto de aportar escalas robustas que permitieran a las instituciones escolares un primer acercamiento a la evaluación de este factor de influencia.

\section{Metodología}

Como parte de la elaboración de un cuestionario se integró casi la totalidad de los ítems que posee la escala de Pont et al. (2009) para la distribución del liderazgo escolar, de la cual se adaptaron algunos vocablos más pertinentes al tipo de destinatario, excluyéndose un ítem que no aplicaba para toda la realidad organizativa de las escuelas primarias en Chile, con lo cual la escala final quedó conformada por nueve ítems. Para la siguiente escala debieron crearse ítems para cada una de las subescalas que conformaban la concepción de estadios de distribución de liderazgo de MacBeath (2011), lo cual generó un total de 45 ítems, reduciéndose cada subescala de ocho a siete ítems. 
Sobre la validez, aparente y de contenido, se generó un perfil de experto en el que estuvieran presentes tanto académicos como profesionales ligados al ámbito directivo escolar. En total, siete personas respondieron los protocolos acerca de la relevancia y pertinencia de las escalas e ítems. Posteriormente, a partir de las valoraciones provistas por los expertos, se conformó una matriz numérica para analizar las valoraciones según el índice de Lawshe (Tristán-López, 2008), con el objeto de contrastar las diferentes opiniones de los expertos en torno a la validez del contenido del instrumento con un indicador estadístico afín. De este modo, se obtuvo una versión piloto que fue administrada a una muestra de directivos docentes superiores pertenecientes a los Consejos Directivos Regionales (conformados por una muestra de directores de escuelas municipales). Ante esto, se generó una segunda versión del cuestionario para ser administrada presencialmente en formato impreso a una muestra de docentes y directivos de 51 escuelas primarias de diversas regiones de Chile. Un total de 723 sujetos respondieron la versión, con una proporción superior a la mitad de la dotación total. Las características generales de la muestra se describen en la Tabla 3.

Tabla 3

Muestra 1: Características de docentes y directivos de 51 escuelas primarias de diferentes regiones de Chile (723)

\begin{tabular}{|c|c|c|c|}
\hline Datos de identificación & \multicolumn{3}{|c|}{ Total y porcentajes (\%) } \\
\hline \multirow[t]{2}{*}{ Género } & Masculino & \multicolumn{2}{|c|}{ Femenino } \\
\hline & $202(27,94)$ & \multicolumn{2}{|c|}{$521(72,06)$} \\
\hline \multirow[t]{2}{*}{ Cargo } & Docente de aula & \multicolumn{2}{|c|}{ Docente directivo } \\
\hline & $598(82,75)$ & \multicolumn{2}{|c|}{$125(17,25)$} \\
\hline \multirow{2}{*}{$\begin{array}{l}\text { Años de experiencia docente } \\
\text { Porcentaje }\end{array}$} & $5-10$ & $10-15$ & $15-25$ \\
\hline & 22,5 & 22 & 30,5 \\
\hline
\end{tabular}

Fuente: Elaboración propia.

La segunda muestra incidental correspondió a 61 escuelas y liceos, cuyos directivos docentes participaron en una versión del Diplomado de Liderazgo Sistémico y Aprendizaje en Red del Centro Líderes Educativos en Chile (Tabla 4). 
178 PERSPECTIVAS DE DIAGNÓSTICO SOBRE LA DISTRIBUCIÓN DEL LIDERAZGO EN ORGANIZACIONES ESCOLARES: UN ANÁLISIS EN DOS DIMENSIONES CLAVE - O. Maureira, S. Garay, L. Ahumada y C. Ascencio

Tabla 4

Muestra 2: Características de docentes y directivos de 61 escuelas y liceos de las regiones chilenas del Biobío y Valparaíso (795)

\begin{tabular}{|c|c|c|c|}
\hline Datos de identificación & \multicolumn{3}{|c|}{ Total y porcentajes (\%) } \\
\hline \multirow{2}{*}{ Género } & Masculino & \multicolumn{2}{|c|}{ Femenino } \\
\hline & $222(27,9)$ & \multicolumn{2}{|c|}{$573(72,1)$} \\
\hline \multirow{2}{*}{ Cargo } & Docente de aula & \multirow{2}{*}{\multicolumn{2}{|c|}{$\frac{\text { Docente directivo }}{120(15,1)}$}} \\
\hline & $675(84,9)$ & & \\
\hline \multirow{2}{*}{$\begin{array}{l}\text { Años de experiencia docente } \\
\text { Porcentaje }\end{array}$} & $5-10$ & $10-15$ & $15-25$ \\
\hline & 32 & 21 & 23 \\
\hline
\end{tabular}

Fuente: Elaboración propia.

\subsection{Análisis comparativos por muestras de las escalas y sus indicadores (ítems)}

Se realizó un análisis de la fiabilidad de las escalas de brechas entre la percepción del estado actual y deseado sobre la base de nueve ítems adaptados y sustentados en Pont et al. (2009), para la muestra de escuelas efectivas y la muestra de participantes de un diplomado en liderazgo sistémico, a partir del coeficiente alfa de Cronbach (1951). Los resultados arrojaron una alta consistencia interna con valores de indices de $\alpha=0,847$ y $\alpha=0,9$ respectivamente.

En cuanto a las dimensiones de patrones o estadios respecto de cómo se distribuye el liderazgo, se encontraron altos valores de fiabilidad en cada una de las dimensiones, en ambas variables, los cuales se detallan a continuación en la Tabla 5.

Tabla 5

Consistencia interna de escalas de estadios o patrones de distribución de liderazgo

\begin{tabular}{lcc}
\hline Dimensiones de tipo de distribución & Muestra 1 $(\mathrm{N}=722)$ & Muestra 2 (N =735) \\
\hline Formal & $\alpha=0,901$ & $\alpha=0,913$ \\
\hline Pragmática & $\alpha=0,79$ & $\alpha=0,813$ \\
\hline Estratégica & $\alpha=0,94$ & $\alpha=0,943$ \\
\hline Incremental & $\alpha=0,944$ & $\alpha=0,95$ \\
\hline Oportuna & $\alpha=0,779$ & $\alpha=0,822$ \\
\hline Cultural & $\alpha=0,917$ & $\alpha=0,923$ \\
\hline
\end{tabular}

Fuente: Elaboración propia. 


\subsection{Análisis Factorial Exploratorio (AFE) de las dimensiones}

Dado el carácter descriptivo y exploratorio del estudio en las dimensiones referidas, se realizó un AFE con el propósito de conocer la agrupación de los ítems en cada dimensión y analizar así su configuración. Para esto, se examinó a través del test de esfericidad de Bartlett, valores significativos en todos los casos $(p<, 001)$ e índices de adecuación muestral satisfactorios basados en Kaiser-MeyerOlkin (KMO) para cada una de las escalas. Luego de verificar tales condiciones mínimas de aplicación del AFE, se realizó un análisis de componentes principales con rotación varimax, cuyas salidas extraídas de SPSS se editaron y resumen a continuación.

\subsubsection{AFE de brecha entre la percepción actual y deseable}

Se realizó un AFE para la brecha entre el liderazgo actual y deseable en ambas bases de datos, el cual se obtuvo a partir de las diferencias entre ambas dimensiones. Se logró una estructura unifactorial para la base de datos de participantes del diplomado de liderazgo sistémico $\left(\mathrm{KMO}=0,925\right.$; Bartlett $\left.\chi^{2}=3.324,806, p<0,01\right)$, la cual explica un $56,41 \%$ de la varianza de los ítems y donde las cargas factoriales son superiores a 0,75, excepto en el caso de la brecha para el ítem nueve. Para el caso de la base de las escuelas ( $\mathrm{N}=722)$, se obtuvo una estructura bifactorial ( $\mathrm{KMO}=0,915$; Bartlett $\left.\chi^{2}=2.798,81, p<0,01\right)$ que explica un $62,72 \%$ de la varianza, pero donde la brecha para el ítem nueve se presenta apartada como una dimensión diferente. En ambos casos es posible observar un comportamiento anormal de este elemento, lo cual sugiere que pudiera tratarse de un aspecto diferente de lo que pretende medir la dimensión en su conjunto, entendiendo esto como la diferencia entre las condiciones de distribución del liderazgo en el colegio y la distribución deseable en cada condición.

2.2.2. Resúmenes de AFE de cada una de las subescalas de las modalidades de distribución de liderazgo

En cada una de las subdimensiones de patrones de distribución de liderazgo afines al establecimiento se realizó un análisis de componentes principales, para poder establecer si estas subescalas constituyen dimensiones unifactoriales. 
Los resultados se exponen a continuación, de acuerdo con cada una de las subdimensiones mencionadas.

\section{a. Distribución formal}

En la dimensión de distribución formal se puede ver en la Tabla 6 una diferencia entre la distribución unifactorial de la muestra 1 y un resultado de dos factores que se aprecia en la muestra 2.

En este segundo caso, los ítems "La estructura jerárquica predomina en la toma de decisiones" y "El liderazgo en esta institución está explícitamente reflejado en las estructuras formales" aparecen separados en un factor diferente.

Tabla 6

Resumen de AFE de escala distribución formal

\begin{tabular}{lcc}
\hline Indicadores & Muestra l $(\mathrm{N}=722)$ & Muestra 2 $(\mathrm{N}=735)$ \\
\hline KMO y $\chi^{2}$ de Bartlett & $\mathrm{KMO}=0,913 ;$ & $\mathrm{KMO}=0,922 ;$ \\
& $\chi^{2}=3366,04, p<0,01$ & $\chi^{2}=3961.67, p<0,01$ \\
\hline $\begin{array}{l}\text { Número de factores y varianza } \\
\text { explicada (VE) }\end{array}$ & 1 factor; VE: $60,37 \%$ & 2 factores; $\mathrm{VE}: 55,23 \%$ \\
\hline
\end{tabular}

Fuente: Elaboración propia.

\section{b. Distribución pragmática}

Para la dimensión evaluada por la subescala de distribución pragmática se puede ver en la Tabla 7 una coherencia entre ambas muestras, lográndose observar resultados de dos factores, en los cuales los ítems "La inmediatez en la asignación de responsabilidades de liderazgo predomina más que la visión" y "Los docentes eluden responsabilidades de liderazgo directivo" se agrupan en un factor diferente del resto de los ítems.

Tabla 7

Resumen de AFE de escala distribución pragmática

\begin{tabular}{lcc}
\hline Indicadores & Muestra $1(\mathrm{~N}=722)$ & Muestra 2 $(\mathrm{N}=735)$ \\
\hline KMO y $\chi^{2}$ de Bartlett & $\mathrm{KMO}=0,834 ;$ & $\mathrm{KMO}=0,852 ;$ \\
& $\chi^{2}=2276,44, p<0,01$ & $\chi^{2}=2333,77, p<0,01$ \\
\hline Número de factores y & 2 factores; VE: $51,04 \%$ y & 2 factores; VE: $51,21 \%$ y \\
varianza explicada (VE) & $17,04 \%$ & $17,32 \%$ \\
\hline
\end{tabular}

Fuente: Elaboración propia. 


\section{c. Distribución estratégica}

Para la dimensión estratégica, en ambas muestras se observa en la Tabla 8 una distribución unifactorial con una elevada varianza explicada por parte del factor extraído.

Tabla 8

Resumen de AFE de escala distribución estratégica

\begin{tabular}{lcc}
\hline Indicadores & Muestra 1 $(\mathrm{N}=722)$ & Muestra 2 $(\mathrm{N}=735)$ \\
\hline KMO y $\boldsymbol{\chi}^{2}$ de Bartlett & $\mathrm{KMO}=0,936 ;$ & $\mathrm{KMO}=0,937 ;$ \\
& $\chi^{2}=4588,3, \mathrm{p}<0,01$ & $\chi^{2}=4992,4, p<0,01$ \\
\hline $\begin{array}{l}\text { Número de factores y varianza } \\
\text { explicada (VE) }\end{array}$ & 1 factor; VE: 70,94\% & 1 factor; VE; 72,07\% \\
\hline
\end{tabular}

Fuente: Elaboración propia.

\section{d. Distribución incremental}

Al igual que en la dimensión anterior, la distribución incremental presenta un único factor con una muy alta varianza explicada sobre los ítems que componen la subescala, tal como se aprecia en la siguiente tabla.

Tabla 9

Resumen de AFE de escala distribución incremental

\begin{tabular}{lcc}
\hline Indicadores & Muestra 1 $(\mathrm{N}=722)$ & Muestra 2 $(\mathrm{N}=735)$ \\
\hline KMO y $\chi^{2}$ de Bartlett & $\mathrm{KMO}=0,919 ;$ & $\mathrm{KMO}=0,918 ;$ \\
& $\chi^{2}=4481,8, p<0,01$ & $\chi^{2}=4942,73, p<0,01$ \\
\hline $\begin{array}{l}\text { Número de factores y } \\
\text { varianza explicada (VE) }\end{array}$ & 1 factor; VE: $74,97 \%$ & 1 factor; VE: 76,96\% \\
\hline
\end{tabular}

Fuente: Elaboración propia.

\section{e. Distribución oportuna}

En el caso de la distribución oportuna, se puede ver una extracción de dos factores diferentes con una varianza total explicada de 63,07\% y $69,77 \%$ respectivamente. En ambas muestras, los ítems "Existen oportunidades en que se nota la ausencia de un líder" y "Se antepone la oportunidad de liderar más que la planificación y la asignación de roles" se perciben como pertenecientes a una dimensión diferente, tal como se muestra en la siguiente tabla. 
182 PERSPECTIVAS DE DIAGNÓSTICO SOBRE LA DISTRIBUCIÓN DEL LIDERAZGO EN ORGANIZACIONES ESCOLARES: UN ANÁLISIS EN DOS DIMENSIONES CLAVE - O. Maureira, S. Garay, L. Ahumada y C. Ascencio

Tabla 10

Resumen de AFE de escala distribución oportuna

\begin{tabular}{lcc}
\hline Indicadores & Muestra $1(\mathrm{~N}=722)$ & Muestra 2 $(\mathrm{N}=735)$ \\
\hline KMO y $\chi^{2}$ de Bartlett & $\mathrm{KMO}=0,85 ;$ & $\mathrm{KMO}=0,877 ;$ \\
& $\chi^{2}=2119,45, p<0,01$ & $\chi^{2}=2972,43, p<0,01$ \\
\hline Número de factores y & 2 factores; VE: $44,89 \%$ y & 2 factores; VE: $49,87 \%$ y \\
varianza explicada & $18,18 \%$ & $19,91 \%$ \\
\hline
\end{tabular}

Fuente: Elaboración propia.

f. Distribución cultural

Por último, la dimensión distribución cultural presenta una estructura unifactorial en ambas muestras, con una varianza explicada de $67,49 \%$ y $68,67 \%$, respectivamente.

Tabla 11

Resumen de AFE de escala distribución cultural

\begin{tabular}{lcc}
\hline Indicadores & Muestra $1(\mathrm{~N}=722)$ & Muestra 2 $(\mathrm{N}=735)$ \\
\hline KMO y $\chi^{2}$ de Bartlett & $\mathrm{KMO}=0,922 ;$ & $\mathrm{KMO}=0,93 ;$ \\
& $\chi^{2}=3473,73, p<0,01$ & $\chi^{2}=3672,62, p<0,01$ \\
\hline $\begin{array}{l}\text { Número de factores y } \\
\text { varianza explicada (VE) }\end{array}$ & 1 factor; VE: $67,49 \%$ & 1 factor; VE: $68,67 \%$ \\
\hline
\end{tabular}

Fuente: Elaboración propia.

2.3. Análisis factorial confirmatorio de la dimensión global estadios o patrones de distribución de liderazgo

Las muestras quedaron compuestas por un total de 722 casos (muestra 1) y 735 casos (muestra 2), eliminándose aquellos cuyas respuestas se hallaban incompletas en al menos una de las dimensiones. Estas muestras fueron examinadas a través de un análisis factorial confirmatorio de la dimensión patrones de distribución de liderazgo, que se configura tal como se ha referido por las subdimensiones formal, pragmática, estratégica, incremental, oportuna y cultural. Así, a través del módulo Amos del Statistical Package for the Social Sciences (Amos, 2012) se probó un modelo consistente en seis subdimensiones relacionadas, con sus respectivos ítems. En ambas muestras se utilizó un procedimiento de estimación de máxima verosimilitud y se usó un conjunto de coeficientes para la evaluación de la bondad de ajuste de los modelos analizados, como se mencionan a continuación. 


\subsubsection{Indicadores de ajuste absoluto}

Estos indicadores no comparan los valores con un indicador específico. Entre ellos se encuentra el valor $\chi^{2}$, que no debiera ser significativo para establecer un adecuado análisis, aunque se ve afectado por el número de sujetos. Por ello, se considera más apropiado el indicador conformado por la razón entre este $\chi^{2}$ sobre los grados de libertad (CMIN/DF), cuyo valor no debiese ser superior a cinco e, idealmente, debe ser menor a tres (los índices de ajuste excelente son inferiores a dos) (Brown, 2006; Hu \& Bentler, 1999). Otro indicador de ajuste absoluto es el índice de bondad de ajuste (GFI), cuyo valor debe ser superior a 0,9. También se reporta la media cuadrática residual (RMR), que debiera tener un valor inferior a 0,05.

\subsubsection{Indicadores de ajuste relativo}

Se compara el ajuste del modelo con un modelo de relaciones nulas, que supone la independencia de los factores. Entre los indicadores utilizados se encuentra el índice de ajuste comparativo (CFI), cuyo valor debiera ser mayor a 0,9 para indicar un ajuste adecuado y el error cuadrático medio de aproximación (RMSEA), el cual debiera obtener un valor inferior a 0,06. Adicionalmente, se obtuvo el índice de Tucker-Lewis, el cual no presenta tendencia a verse afectado por el tamaño de la muestra.

Los resultados son muy similares en ambas muestras y se observa un ajuste que es adecuado en la mayor parte de los indicadores, salvo el de bondad de ajuste GFI (donde debiese ser superior a 0,9) y RMSEA, el cual posee valores de ajuste que debieran ser inferiores a 0,6. Se propuso, entonces, un modelo sin aquellos ítems que presentaban indicadores de ajuste bajo y varianzas explicadas bajo 0,2 .

Tabla 12

Resultados para la muestra participantes del diplomado en liderazgo sistémico, con 735 casos

\begin{tabular}{lcccccc}
\hline Modelo & CMIN/DF & GFI & CFI & RMR & RMSEA & TLI \\
\hline Inicial & 3,675 & 0,805 & 0,915 & 0,040 & 0,06 & 0,909 \\
\hline Final & 3,3 & 0,853 & 0,943 & 0,027 & 0,056 & 0,939 \\
\hline
\end{tabular}

Fuente: Elaboración propia. 
184 PERSPECTIVAS DE DIAGNÓSTICO SOBRE LA DISTRIBUCIÓN DEL LIDERAZGO EN ORGANIZACIONES ESCOLARES: UN ANÁLISIS EN DOS DIMENSIONES CLAVE - O. Maureira, S. Garay, L. Ahumada y C. Ascencio

Tabla 13

Resultados para la muestra de escuelas efectivas, con 722 casos

\begin{tabular}{lcccccc}
\hline Modelo & CMIN/DF & GFI & CFI & RMR & RMSEA & TLI \\
\hline Inicial & 3,655 & 0,81 & 0,905 & 0,043 & 0,061 & 0,899 \\
\hline Final & 3,406 & 0,849 & 0,933 & 0,032 & 0,058 & 0,928 \\
\hline
\end{tabular}

Fuente: Elaboración propia.

Al realizar el análisis sin los ítems antes señalados, se pueden encontrar indicadores de ajuste ligeramente superiores, en especial en el caso de RMSEA y RMR, lo cual puede deberse a un mejor ajuste de la estructura factorial. No obstante, la marginalidad de las diferencias entre ambos modelos desaconseja perder ítems que pueden ser especialmente útiles en la medición de las dimensiones deseadas.

\section{Conclusiones}

Si bien en esta última década existe una importante discusión teórica en cuanto al enfoque de liderazgo en las organizaciones escolares y, en particular, lo que dice relación con el denominado liderazgo distribuido, en su mayoría proviene del ámbito anglosajón $y$, en el caso de las investigaciones nacionales, gran parte de los estudios corresponden a investigaciones de casos únicos, o bien, de casos múltiples de carácter cualitativos o cuantitativos, pero con una muestra de escuelas muy pequeña. Solo uno de los estudios chilenos (López y Gallegos, 2017) expuestos posee un tamaño de muestra, aunque con criterio incidental que es sustantivo en cuanto a magnitud.

La gran mayoría de las investigaciones, directa o indirectamente, vincula las perspectivas de liderazgo distribuido con procesos clave o con indicadores de resultados escolares. Asimismo, fundamentan sus opciones teóricas en función de referentes anglosajones similares. Esto último se refleja — dado lo situacional del fenómeno de liderazgo-, en cierta escases de referentes teóricos propios de la idiosincrasia organizativa escolar en la región. En este sentido, los estudios nacionales revisados en la introducción de este trabajo representan un aporte para comprender el desarrollo del liderazgo distribuido en distintos contextos a nivel nacional. De esta forma, el presente estudio busca contribuir a incrementar la literatura de liderazgo distribuido 
en Chile y a complementar los estudios internacionales de Pont et al. (2009) y MacBeath (2011) que han aportado las dimensiones aquí revisadas y que ofrecen una perspectiva útil para generar estructuras más participativas que contribuyan al mejoramiento escolar y al desarrollo de procesos más innovadores.

Por su parte, en relación con las diferentes características y acepciones con las que la literatura científica connota al liderazgo distribuido y su operacionalización, de cara a su evaluación o diagnóstico, fue posible encontrar dos perspectivas de aplicación en el contexto chileno: la de Hulpia et al. (2009) empleada en dos estudios (Derby, 2017; López y Gallegos, 2017) y la de Davis (2009), usada en un estudio comparativo en escuelas municipales de una ciudad del norte de Chile (Rojas-Andrade et al., 2018). Ambas perspectivas definidas multidimensionalmente por medio de inventarios y con cierta tendencia a privilegiar entre sus dimensiones la valoración de liderazgo distribuido por medio del apoyo o supervisión del director o directora. En cambio, uno de los criterios que da más sentido a las dos dimensiones defendidas, descritas y analizadas en el presente trabajo fue el de relevar, como unidad de análisis, la escuela o el centro escolar, reconociendo que tanto los directivos como su equipo son agentes clave en la distribución de liderazgo, y cuya transferencia de poder está mediada por aspectos culturales, históricos y normativos que la condicionan. Así, las dos perspectivas de liderazgo distribuido que fueron adaptadas y contextualizadas a la realidad organizativa escolar para este estudio, no mencionan directamente al director o directora ni a sus equipos directivos, focalizándose más en la escuela como organización.

Desde el punto de vista de los análisis realizados, así como de su pertinencia para la práctica organizativa de las escuelas y liceos en Chile, ambas escalas y en muestras diferentes presentan una alta consistencia interna, es decir, son estables en su medida. Así, en relación con los análisis factoriales exploratorios cabe precisar que la primera escala aporta con un indicador de medida que va más allá de lo planteado por Pont et al. (2009), pues se provee de un constructo que captura, a modo de percepción, la medida de la brecha entre lo percibido actual y deseado sobre la distribución del liderazgo. Se 
186 PERSPECTIVAS DE DIAGNÓSTICO SOBRE LA DISTRIBUCIÓN DEL LIDERAZGO EN ORGANIZACIONES ESCOLARES: UN ANÁLISIS EN DOS DIMENSIONES CLAVE - O. Maureira, S. Garay, L. Ahumada y C. Ascencio

ofrece, de esta manera, un enfoque de diagnóstico plausible tanto como indicador agregado o desagregado. Respecto del análisis de dicha escala —que buscó establecer la percepción de brechas entre el estado actual y lo deseado manifestadas en cuanto a oportunidades, promoción y estructuras de desarrollo de liderazgos al interior de los establecimientos y la percepción de convicción que existe acerca de si el liderazgo compartido favorecería los aprendizajes— se observó una estructura mayoritariamente de un solo factor. Esto explica en promedio poco más de la mitad de la varianza. No obstante, se constató, en ambas comparaciones de muestras que, precisamente, el ítem referido a las convicciones de que el liderazgo compartido favorecería el aprendizaje es más bien concebido como un aspecto diferente de medición de los restantes ocho ítems que conforman la escala, lo que sugeriría extraerlo de dicha posición e incorporarlo como un ítem de criterio de validez interna.

Por su parte, la segunda perspectiva de liderazgo distribuido, cuyo propósito es indagar en cuanto a patrones o estadios de modalidades de distribución de liderazgo, sustentada por MacBeath (2011), se posiciona teóricamente de manera mucho más exhaustiva y rica para captar la construcción institucional predominante de la distribución del liderazgo, respecto de otros marcos conceptuales como el de Gronn (2002) o Leithwood (2009).

En cuanto al análisis factorial exploratorio sobre patrones de distribución de liderazgo, solo tres escalas tienen una estructura unifactorial, donde las puntuaciones se comportan de manera muy similar en ambas muestras. Con esto, se destaca la alta varianza explicada del único factor por los ítems de las escalas de distribución estratégica, incremental y cultural, respectivamente. En cambio, en las escalas de distribución formal y oportuna emerge un segundo factor que complejiza las atribuciones de la medida del factor por sus ítems. La única escala que ofrece un comportamiento distinto, según comparativo de muestra, es la escala sobre distribución formal.

Con todo, se logró justificar, describir, analizar y exponer desagregadamente dos dimensiones plausibles de sustentar de modo operacional para un primer acercamiento al diagnóstico del 
liderazgo distribuido en las instituciones escolares, pues el cúmulo teórico de argumentación sugiere que es una condición fundamental para cultivar y anidar mayores niveles de confianza, compromiso organizacional e implicación para elevar el desarrollo de capacidades escolares e incrementar el capital social y decisional en las escuelas.

Finalmente, se debe señalar que ambas dimensiones tienen un sustento relevante para la profundización en la medición de la distribución del liderazgo, las que al estar integradas en formato de cuestionario permiten una fácil administración para un primer diagnóstico que suscite la reflexión profesional tanto docente como directiva, en prácticas de liderazgo escolar, sobre propuestas de acción más coherentes con la realidad organizativa, de cara a la mejora de diversos aprendizajes requeridos en las instituciones escolares.

\section{Referencias}

Ahumada, L. (2010). Liderazgo distribuido y aprendizaje organizacional: tensiones y contradicciones de la actual Reforma educacional. Psicoperspectivas. Individuo y Sociedad, 9(1), 111-123. https://doi. org/10.5027/psicoperspectivas-vol9-issuel-fulltext-99

Ahumada, L., Castro, S., y Maureira, O. (2018). Modelo para el fortalecimiento del liderazgo distribuido en escuelas y liceos mediante indagación colaborativa. Nota Técnica $N^{o}$ 9. Recuperado de https://www.lidereseducativos. cl/wp-content/uploads/2019/02/NT9_MODELO-PARA-ELFORTALECIMIENTO-DEL-LIDERAZGO-DISTRIBUIDO_26-12-18. pdf

Ahumada, L., González, Á., y Pino, M. (2016). Redes de mejoramiento escolar: ¿Por qué son importantes y como las apoyamos? Documento de trabajo $N^{\circ}$ 1. Recuperado de https://www.lidereseducativos.cl/recursos/redespara-el-mejoramiento-escolar/

Amos, I. S. (2012). IBM SPSS Amos. IBM Software Business Analytics, YTD03114-U, 1-8.

Bellei, C., Morawietz, L., Valenzuela, J., y Vanni, X. (2015). Nadie dijo que era fácil. Escuelas efectivas en sectores de pobreza, diez años después. Santiago de Chile: LOM Ediciones.

Bellei, C., Valenzuela, J., Vanni, X., y Contreras, D. (2014). Lo aprendí en la escuela. ¿Cómo se logran procesos de mejoramiento escolar? Santiago de Chile: LOM Ediciones. 
188 PERSPECTIVAS DE DIAGNÓSTICO SOBRE LA DISTRIBUCIÓN DEL LIDERAZGO EN ORGANIZACIONES ESCOLARES: UN ANÁLISIS EN DOS DIMENSIONES CLAVE - O. Maureira, S. Garay, L. Ahumada y C. Ascencio

Bennett, N., Wise, C., Woods, P., \& Harvey, J. (2003). Distributed leadership: A review of literature. London: National College of School Leadership.

Bolden, R. (2011). Distributed leadership in organizations: A review of theory and research. International Journal of Management Reviews, 13(3), 251269. https://doi.org/10.1111/j.1468-2370.2011.00306.x

Bolívar, A. (2012). Politicas actuales de mejora y liderazgo educativo. Madrid: Ediciones Aljibe.

Brown, T. (2006). Confirmatory factor analysis for applied research. New York, London: The Guilford Press.

Cronbach, L. (1951). Coefficient alpha and the internal structure of tests. Psychometrika, 16(3), 297-334. https://doi.org/10.1007/bf02310555

Davis, M. (2009). Distributed leadership and school performance (Tesis de doctorado inédita). George Washington University, Washington DC., USA.

Derby, F. (2017). Liderazgo distribuido en procesos de gestión curricular (Tesis doctoral inédita). Pontificia Universidad Católica de Chile, Santiago, Chile.

Engeström, Y. (2001). Los estudios evolutivos del trabajo como punto de referencia de la teoría de la actividad: el caso de la práctica médica de la asistencia básica. En S. Chaiklin y J. Lave (Comps.), Estudiar las prácticas: perspectivas sobre actividad y contexto (pp. 78-118). Buenos Aires: Amorrortu Editores.

Fernández, I. y Zambrano, R. (2018). Liderazgo efectivo para el alto desempeño. Santiago de Chile: Editorial Empresa Activa.

García Torres, D. (2018). Distributed leadership and teacher job satisfaction in Singapore. Journal of Educational Administration, 56(1), 127-142. https://doi.org/10.1108/jea-12-2016-0140

Gronn, P. (2002). Distributed leadership as a unit of analysis. The Leadership Quarterly, 13(4), 423-451. https://doi.org/10.1016/s10489843(02)00120-0

Harris, A. (2012). Liderazgo y desarrollo de capacidades en la escuela. Santiago de Chile: Centro de Innovación en Educación de Fundación Chile.

Harris, A. (2014). Distributed leadership matters: Perspectives, practicalities, and potential. California: Corwin.

Hu, L. \& Bentler, P. (1999). Cutoff criteria for fit indexes in covariance structure analysis: Conventional criteria versus new alternatives. Structural Equation Modeling: A Multidisciplinary Journal, 6(1), 1-55. https://doi.org/10.1080/10705519909540118 
Hulpia, H., Devos, G., \& Rosseel, Y. (2009). Development and validation of scores on the distributed leadership inventory. Educational and Psychological Measurement, 69(6), 1013-1034. https://doi. org/10.1177/0013164409344490

Hulpia, H., Devos, G., \& Van Keer, H. (2011). The relation between school leadership from a distributed perspective and teachers' organizational commitment: Examining the source of the leadership function. Educational Administration Quarterly, 47(5), 728-771. https://doi. org/10.1177/0013161x11402065

Hutchins, E. (1995). How a cockpit remembers its speed. Cognitive Science, 19(3), 265-288. https://doi.org/10.1207/s15516709cog1903_1

Larrain, F. (2017). The changes in relational trust during the first year of a distributed leadership implementation: A descriptive study on the changes of trust among distributed leadership teams (Disertación doctoral). Recuperado de ProQuest Dissertations \& Theses Global. (Acceso ${ }^{\circ}$ 10287344).

Leithwood, K. (2009). ¿Cómo liderar nuestras escuelas? Aportes desde la investigación. Santiago de Chile: Área de Educación Fundación Chile.

Ley No 20.501 Calidad y Equidad de la Educación. Diario Oficial de la República de Chile, Santiago, Chile, 26 de febrero de 2011. Recuperado de https://www.leychile.cl/Navegar?idNorma=1022346

López, P. y Gallegos, V. (2017). Liderazgo distribuido y aprendizaje de la matemática en escuelas primarias: el caso de Chile. Perfiles Educativos, 39(158), 112-129. https://doi.org/10.22201/ iisue.24486167e.2017.158.58786

López, P. (2013). Fundamentos epistemológicos del liderazgo distribuido: el caso de la investigación en educación. Cinta de Moebio, 47, 83-94. https://doi.org/10.4067/s0717-554x2013000200003

López-Yáñez, J. (2012). Visiones que deslumbran. El declive del liderazgo transformacional. Organización y Gestión Educativa, 95(3), 8-11.

MacBeath, J. (2005). Leadership as distributed: A matter of practice. School Leadership and Management, 25(4), 349-366. https://doi. org/10.1080/13634230500197165

MacBeath, J. (2011). Liderar el aprendizaje dentro y fuera de la escuela. Santiago de Chile: Área de Educación Fundación Chile.

Maureira, O. (2013). Distribución del liderazgo en escuelas de Chile: Un estudio exploratorio. Revista Intersecciones Educativas, 3(3), 1-17. 
190 PERSPECTIVAS DE DIAGNÓSTICO SOBRE LA DISTRIBUCIÓN DEL LIDERAZGO EN ORGANIZACIONES ESCOLARES: UN ANÁLISIS EN DOS DIMENSIONES CLAVE - O. Maureira, S. Garay, L. Ahumada y C. Ascencio

Montecinos, C., Aravena, F., y Tagle, R. (2016). Liderazgo escolar en los distintos niveles del sistema: Notas técnicas para orientar sus acciones. Recuperado de https://www.lidereseducativos.cl/wp-content/uploads/2017/01/ Liderazgo-Escolar-en-los-Distintos-Niveles-del-Sistema-LIDERESEDUCATIVOS.pdf

Morales, P., Urosa, B., y Blanco, Á. (2003). Construcción de escalas de actitudes tipo Likert. Madrid: Editorial La Muralla.

Organisation for Economic Co-operation and Development, OECD. (2014). TALIS 2013 Results: An international perspective on teaching and learning. Paris: Autor.

Pascual, J., Larraguibel, D., Zenteno, D., y Guarda, F. (2016). Liderazgo escolar en tiempos de crisis. El caso de dos liceos del centro sur de Chile después del 27F. REICE. Revista Iberoamericana sobre Calidad, Eficacia y Cambio en Educación, 14(2), 45-62. https://doi.org/10.15366/ reice2016.14.2.003

Pont, B., Nusche, D., y Moorman, H. (2009). Mejorar el liderazgo escolar, Volumen 1: Política y práctica. Paris: OECD Publishing.

Real Academia Española, RAE. (2019). Diccionario de la lengua española. Recuperado de https://dle.rae.es/?id=DzNTv6G

Rojas-Andrade, R., Prosser, J., y Bonilla, N. (2018). Distribuir el liderazgo para mejorar la calidad de la educación. CASTALIA, 4(3), 38-49. https:// doi.org/10.25074/07198051.30.921

Sans-Martín, A., Guàrdia, J., y Triadó-Ivern, X. (2016). El liderazgo educativo en Europa: una aproximación transcultural. Revista de Educación, 371, 83-106. Recuperado https://sede.educacion.gob.es/publiventa/ el-liderazgo-educativo-en-europa-una-aproximacion-transcultural-educational-leadership-in-europe-a-transcultural-approach/ investigacion-educativa/20644

Senge, P., Ross, R., Smith, B., Roberts, C., y Kleiner, A. (1995). La quinta disciplina en la práctica: estrategias para construir la organización abierta al aprendizaje. Madrid: Ediciones Juan Gránica.

Sergiovanni, T. (1984). Leadership and excellence in schooling. Educational Leadership, 41(5), 4-13.

Spillane, J. (2006). Distributed leadership. San Francisco: Jossey-Bass.

Spillane, J. \& Healey, K. (2010). Conceptualizing school leadership and management from a distributed perspective: An exploration of some study operations and measures. The Elementary School Journal, 111(2), 253-281. https://doi.org/10.1086/656300 
Spillane, J., Camburn, E., Pustejovsky, J., Stitziel, A., \& Lewis, G. (2008). Taking a distributed perspective: Epistemological and methodological tradeoffs in operationalizing the leader-plus aspect. Journal of Educational Administration, 46(2), 189-213. https://doi. org/10.1108/09578230810863262

Spillane, J., Halverson, R. y Diamond, J. (2001). Investigating school leadership practice: A distributed perspective. Educational Researcher, 30(3), 23-28. https://doi.org/10.3102/0013189x030003023

Spillane, J., Halverson, R., \& Diamond, J. (2004). Towards a theory of leadership practice: A distributed perspective. Journal of Curriculum Studies, 36(1), 3-34. https://doi.org/10.1080/0022027032000106726

Timperley, H. (2005). Distributed leadership: Developing theory from practice. Journal of Curriculum Studies, 37(4), 395-420. https://doi. org/10.1080/00220270500038545

Tristán-López, A. (2008). Modificación al modelo de Lawshe para el dictamen cuantitativo de la validez de contenido de un instrumento objetivo. Avances en Medición, 6(1), 37-48.

Weinstein, J. (2016). Liderazgo educativo en la escuela. Nueve miradas. Santiago de Chile: Ediciones Universidad Diego Portales.

Zener, K. (2011). Understanding principals' and lead teachers' perceptions of distributed leadership in three high-performing public high schools: An exploratory multiple case study (Tesis de doctorado inédita). California State University. Fullerton, USA.

Recibido: 15/06/2019

Aceptado: 18/10/2019 